NUCLEIC ACIDS

\section{Collapsible DNA}

from our Molecular Biology Correspondent

WITH a metre or so length of DNA in each of one's cell, it is fitting that one should from time to time ponder the question of how this relates to the wellknown hydrodynamic rigidity of the thread of life, once this is out of the living cell and in the ultracentrifuge cell, where in the view of many people it properly belongs. Its properties, one might hazard, are modified by the basic proteins in which, in higher organisms, it is packaged. In other surroundings, such as phage heads, such a facile evasion will not do, and this is the starting point of Lerman's efforts (Proc. US Nat. Acad. Sci., 68, $1886 ; 1971)$ to find conditions in which native DNA will pack into a compact form, as it does in the phage.

In dilute salt solutions DNA is a rather stiff "worm-like coil". In the presence of high concentrations of neutral, unreactive polymers, Lerman reasoned, an effective phase separation, such as occurs macroscopically in mixtures of mutually repelling polymer species in solution, could be achieved by a collapse of this extended structure into a compact domain. Moreover, if the intermolecular forces in such a system are indeed predominantly repulsive, one would not expect that this would have to be accompanied by denaturation of the double helix.

The argument was evidently sound, for in the presence of the neutral polymer, polyethylene oxide ('Carbowax'), and at a sufficient salt concentration, presumably to minimize internal repulsions, a dramatic collapse of the DNA structure occurs. T4 phage DNA, which normally sediments at about 80 S, now forms a broad band in a sucrose$\mathrm{D}_{2} \mathrm{O}$ gradient, centred at about $300 \mathrm{~S}$, and extending to $500-600 \mathrm{~S}$. With T7 DNA the results are qualitatively similar: from a normal sedimentation rate of $62 \mathrm{~S}$, it changes to give a peak at $200-250 \mathrm{~S}$, trailing out to $400 \mathrm{~S}$. The transition to the compact state occurs within a critical range of neutral polymer concentration and of sodium chloride (between 0.2 and $0.3 \mathrm{M}$ ). The polyethylene oxide itself undergoes no detectable change, as judged by its viscosity, in this ionic strength range, and moreover another neutral polymer, polyvinylpyrrolidone, will serve iust as well. A striking demonstration of the existence of a threshold polymer concentration was achieved by sedimenting the DNA through a sucrodensity gradient containing an inverse concentration gradient of polyethylene oxide. On encountering a low polymer concentration the DNA reverts to the slowly sedimenting form, so that the back of the sedimenting zone catches up the front, and practically stops.

Examination of the buoyant density of the compact DNA after sedimentation excludes any question of denaturation. The sedimentation patterns are independent of DNA concentration, so that one may take it that the rapidly sedimenting form is not aggregated material. The high partial specific volume of the neutral polymer means that the formation of a complex with DNA, even with a large excess of polymer, would make little impression on the sedimentation coefficient of the DNA. Moreover, a similar effect is exerted by polyacrylic acid, which it would be hard to envisage forming a complex with DNA. It thus seems justified to infer that a true compact form is indeed generated. One may calculate sedimentation coefficients of the DNA molecule in various forms, and one may with reasonable certainty exclude supercoiling, which would change the sedimentation rate far too little, and a range of other possibilities. A compact sphere would sediment too rapidly, but an ellipsoid of axial ratio about $7: 1$ would give a result of the right order, and so would a platelet made up of double-helical stretches of DNA some $160 \AA$ long folded concertinawise on each other.

So far as theory for phase relations can be applied, it is by no means inconsistent with a microscopic phase separation in the DNA-polymer system. Lerman suggests that the intracellular environment, pullulating as it is with other macromolecules, may be not unlike his model system. The implications of the ready transition from a stiff to a highly folded form in relation to the structure of the double helix will have to be carefully considered.

\title{
Reverse Transcriptase Initiates on RNA Primer
}

IN next Wednesday's Nature New Biology, Baltimore and four of his colleagues report that reverse transcriptase requires an RNA primer to initiate the synthesis of a DNA chain, which is covalently bound to the primer RNA. They describe a series of experiments which prove beyond doubt that the enzyme initiates DNA synthesis by adding a deoxynucleotide to the $3^{\prime}$ hydroxyl group of the primer. In short, the mechanism of DNA synthesis by reverse transcriptase seems to be essentially identical to that of the Kornberg DNA polymerase I of Escherichia coli, except the reverse transcriptase can use an RNA primer and an RNA template. The similarities between the two enzymes are indeed so great that Verma et al. even suggest that an RNA molecule might serve as the initiator or primer for DNA synthesis in $E$. coli by enzymes such as DNA polymerase I.

The first real clue that reverse transcriptase might require a primer RNA came from experiments with exogenous templates. Baltimore and Smoler found that although the enzyme often uses exogenous templates more efficiently than the endogenous tumour virus genome, it does so only when a primer, which can be as short as a tetranucleotide, is present; the product made comprises the new DNA chain physically attached to the primer. Having made these observations Baltimore's group asked the obvious and important question-does the enzyme when it transcribes the endogenous tumour virus genome require a primer and if so what is the primer?

They first noticed that the 20 minute product of the endogenous polymerase reaction with murine leukaemia virus particles was a single stranded DNA with a density slightly greater than expected. By treating this DNA with ribonuclease or alkali its density was reduced to that of single stranded DNA. Turning to avian myeloblastosis virus particles, which can be had in large amounts, they analysed the product DNA made after 2 to 30 minutes by the endogenous reaction. As the reaction proceeds the density of the DNA product shifts from that of a $1: 1$ RNA.DNA duplex to close to that of single stranded DNA.

After a series of treatments which hydrolyse RNA all the reaction products assumed the density of single stranded DNA. Using purified avian myeloblastosis virus enzyme and isolated avian myeloblastosis virus RNA instead of the virus particles, they obtained essentially identical results. And experiments with the inhibitor of reverse transcriptase, dideoxythymidine triphosphate, which have yet to be published by Baltimore's group apparently confirm that DNA synthesis is initiated by the attachment of the first deoxynucleotide to the $3^{\prime}$ hydroxyl group of the primer.

But what acts as primer in the endogenous reaction? It could of course be that the $3^{\prime}$ end of the $60-70 \mathrm{~S}$ viral RNA genome forms a hair pin bend; alternatively small RNA molecules might associate with the 60-70S RNA. Small RNAs, including transfer RNAs, are present in tumour virus particles and Baltimore's group favour the idea that these act as primers, not least because many DNA molecules are made per viral genome. 